\title{
New records of lichens and allied fungi from Vodlozersky National Park within Arkhangelsk Region (NW Russia)
}

\section{Viktoria N. Tarasova ${ }^{1}$, Tatiana N. Pystina ${ }^{2}$, Vera I. Androsova ${ }^{1}$, Angella V. Sonina ${ }^{1}$, Andrei A. Valekzhanin ${ }^{3}$, Liudmila A. Konoreva ${ }^{4,5}$}

\author{
${ }^{1}$ Department of Botany and Plant Physiology, Petrozavodsk State University, 33 Lenin str., 185910 Petrozavodsk, Russia. \\ E-mails: tarasova1873@gmail.com, vera.androsova28@gmail.com, angella_sonina@mail.ru \\ ${ }^{2}$ Department of Flora and Vegetation of the North, Institute of Biology of Komi Scientific Centre of the Ural Branch of \\ the Russian Academy of Sciences, 28 Kommunisticheskaya str., 167982 Syktyvkar, Russia. \\ E-mail: t.pystina@ib.komisc.ru \\ ${ }^{3}$ Department of Botany, Northern (Arctic) Federal University, 17 Severnaya Dvina emb., 163002 Arkhangelsk, Russia. \\ E-mail: valekzhanin13@gmail.com \\ ${ }^{4}$ Laboratory of Lichenology and Bryology, Komarov Botanical Institute of RAS, 2 Professor Popov str., \\ 197376 St. Petersburg, Russia. \\ ${ }^{5}$ Polar-alpine Botanical Garden-Institute, 184250 Kirovsk Murmansk Region. \\ E-mail: ajdarzapov@yandex.ru
}

\begin{abstract}
The paper presents the results of the ongoing research of lichen diversity in Arkhangelsk Region of Russia, in Vodlozersky National Park which is the largest protected area in the territory of NW Russia. In total, 155 species of lichens and allied fungi are recorded for the first time for the Arkhangelsk part of the Vodlozersky National Park, and 69 species - for the whole mainland area of Arkhangelsk Region.
\end{abstract}

Keywords: lichen diversity, new lichen records

\section{INTRODUCTION}

Nowadays when the environment has been strongly transformed, nature protected territories play the significant role in the conservation and maintenance of main typical landscapes and biodiversity in natural state.

Vodlozersky National Park is one of the largest $(468,300 \mathrm{ha})$ protected areas in the territory of European Russia, as well as in whole Europe. The park is located within two administrative regions: Arkhangelsk Region $(337,600$ ha) and Republic of Karelia (130,600 ha). The territory of the park is extended from north to south and covered by different types of communities. Northern (Arkhangelsk) part of the Park represents the territory of northern boreal subzone with old-growth forests, high habitat heterogeneity and climatic features which are related to the vicinity of the White Sea. The low-mountain ridge Vetreny Poyas (in engl. Windy belt) located here is assumed as the edge of Baltic shield and transition zone to European Plain.

Furthermore, in such environmental conditions (northern boreal zone, relief changes, vicinity of the sea), old-growth forests which have never been subjected to clear-cuts (with the exception of selective cuttings near the villages which have been abandoned at present time) occur. Thus, the unique nature communities in this territory have mainly determined the status of Vodlozersky National Park as UNESCO Biosphere Reserve since 2001.

Despite more than 25 years of lichenological studies in Vodlozersky National Park, only scattered data can be found in various sources which mainly describe the lichen diversity of the Karelian part (Tarasova \& Stepanova, 2001; Tarasova \& Stepanchikova, 2016; Tarasova et al., 2016, 2017; Ignatenko \& Tarasova, 2017; Androsova et al., 2018). Description of the history of lichenological studies in this territory is provided in Tarasova et al. (2016). The lichen inventory of the Arkhangelsk part of the Park has recently begun (Tarasova et al., 2016). In general, the lichen diversity of the Arkhangelsk Region is still poorly known compared to other regions of Russia. 
Long-term lichen inventories in such an etalon territory as the Vodlozersky National Park increase our knowledge of biodiversity as the Park contributes significantly to the maintaining of species pool in the region where most forests have been cut down during the last 100 years. The present study focuses on the new records of lichens and allied fungi for the Arkhangelsk part of Vodlozersky National Park and the whole mainland area of the Arkhangelsk Region.

\section{MATERIALS AND METHODS}

The study area is located in the north-western part of the Arkhangelsk Region, in the northern part of Vodlozersky National Park (Fig. 1.), about $40-70 \mathrm{~km}$ from the coastline of Onega Bay of the White Sea. The environmental characteristics of the area were described in detail in our previous papers (Tarasova et al., 2015, 2016).

The field work was carried out by the first five authors during six expeditions in 2010-2018. Specimens were collected in different types of communities from swamps and old-growth forests (with age exceeding 200 years) to overgrown fields around abounded villages. The main phorophytes were spruce (Picea obovata Ledeb. and P. abies (L.) Karst.), birch (Betula pubescens Ehrh.), aspen (Populus tremula L.), rowan (Sorbus aucuparia L.), willow (Salix caprea L.) and pine (Pinus sylvestris L.).

The collected material, a total of $\sim 1800$ lichen specimens, was identified using standard microscopic technique and spot tests. The specimens of the genus Cladonia and sterile crustose lichen species were identified using thin-layer chromatography (TLC) in the Laboratory of Experimental Botany of Petrozavodsk State University (Petrozavodsk) and high performance thin-layer chromatography (HPTLC) in the Laboratory of Lichenology and Bryology of Komarov Botanical Institute of RAS (St. Petersburg), implementing solvent systems A, B and C (Orange et al., 2001). The specimens of Peltigera were confirmed by Orvo Vitikainen (Botanical Museum, University of Helsinki). The cited specimens are deposited in the Herbarium of Petrozavodsk State University (PZV) and Komi Research Centre (SYKO).

\section{LIST OF SPECIES}

Taxa are arranged in alphabetical order; nomenclature of lichens, lichenicolous and nonlichenized fungi follows Nordin et al. (2011). For each species the localities, habitat types and substrates are listed. Lichen substances are given for TLC-analyzed species.

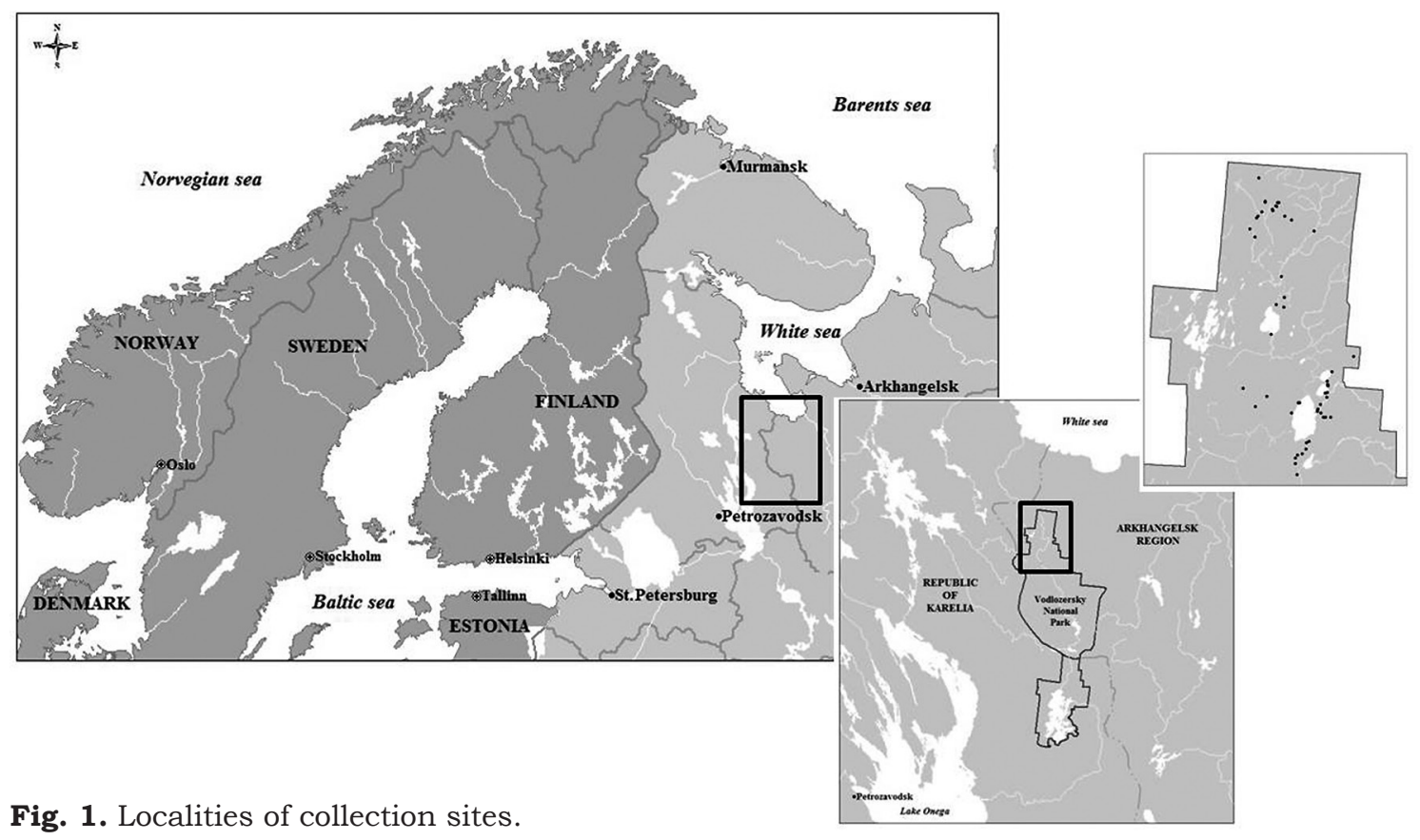


Abbreviations and symbols: \# - lichenicolous fungi; + - non-lichenized fungi; ! - new species for mainland area of Arkhangelsk Region. Species included in the Red Data Book of Arkhangelsk region (2008) are marked as RA. The names of the main collectors in the species list are abbreviated as follows: AS - Angella Sonina, AV - Andrey Valekzhanin, ECh - Elena Churakova, TP - Tatiana Pystina, VA - Vera Androsova, VT - Viktoria Tarasova.

Abbreviations of forest communities and other habitats:

Ssph-spruce swamp forest of Sphagnum mosses type;

Sher - spruce forest of wet herb-rich type;

Smyr - spruce forest of Vaccinium myrtillusfeather mosses type;

Sroc - spruce rocky forest of Vaccinium myrtillus-feather mosses type;

Sbir- mixed spruce-birch rocky forest of lichenfeather mosses type;

Spin - mixed spruce-pine forests of Vaccinium myrtillus-feather mosses type;

Psph - pine forest of Sphagnum mosses type;

Pvit - pine forest of Vaccinium vitis-idaea-feather mosses type on top of the ridge;

Plich - pine forest of Calluna vulgaris-lichens type on the ridge;

Pmyr - pine forests of Vaccinium myrtillusfeather mosses type;

Proc - pine rocky forest of Vaccinium myrtillusfeather mosses type;

Pspr - mixed pine-spruce forest of Sphagnum mosses type;

Psb - mixed pine-spruce-birch forest of Sphagnum mosses type;

Bsph - birch forest of Sphagnum mosses type;

Bher - birch herb forest of feather mosses type;

Basp - mixed birch-aspen forest of feather mosses type;

Aher - aspen herb-rich forest of feather mosses type;

Sw - Sphagnum swamp with pine;

FR - old forest road;

$\mathrm{AbVi}$ - abandoned village, meadows, freestanding trees;

Rsh - river bank;

$\mathrm{R}$ - rocks, boulders.

AlyXORIA VARIA (Pers.) Ertz \& Tehler - on bark of old rowan, 6319'19.6”N, 3643'12.2”E, Spin, 04.07.2016, leg. TP, SYKO.
Amandinea Punctata (Hoffm.) Coppins \& Sheard. - on bark of willow, worked timber, $63^{\circ} 19^{\prime} 39.3^{\prime \prime} \mathrm{N}$ 36²'15.6"E, AbVi, RSh, 15.06.2011, leg. VT, PZV; 6319'45.9”N 3642'17.5”E, AbVi, 01.07.2016, leg. TP, SYKO.

ARTHONIA DISPUNCTA Nyl. - on smooth bark of willow, 63³0'51.1"N 36³7'28.2”E, Sbir, Sroc, leg. VT, 09.06.2018, PZV; on bark of aspen, $63^{\circ} 30^{\prime} 7.0^{\prime \prime} \mathrm{N} 36^{\circ} 38^{\prime 20.0 ”} \mathrm{E}$, Spin, 24.06.2018, leg. AV, PZV.

ARTHONia PATELlULATA Nyl. - on smooth bark of aspen, 6330'51.1'N 36³7'28.2"E, Sbir, Sroc, 08.06.2018, leg. VT, PZV.

ARthonia Radiata (Pers.) Ach. - on bark of aspen, $63^{\circ} 20^{\prime} 53.6^{\prime \prime N ~ 36 ³ 3 ' 18.5 ” ~ E, ~ S m y r t, ~ 14.06 .2011, ~}$ leg. VT, PZV.

ARTHOPYRENIA GRISEA (Schleich. ex Schaer.) Körb. - on decaying wood, 6330'51.1"N 36 37'28.2” $\mathrm{E}$, E, Sbir, Sroc, 06.06.2018, leg. VT, PZV.

+ARTHOTHELIUM SCANDINAVICUM Th. Fr. - on bark of standing deadwood of spruce, 63 $30^{\prime} 51.1^{\prime \prime} \mathrm{N}$ 36³7’28.2”E, Sbir, Sroc, 06.06.2018, leg. VT, PZV.

! Athallia holocarpa (Hoffm.) Arup et al. - on bark of aspen, $63^{\circ} 19^{\prime} 45.9^{\prime \prime} \mathrm{N} 36^{\circ} 42^{\prime} 17.5^{\prime \prime} \mathrm{E}$, $63^{\circ} 19^{\prime} 33.4^{\prime \prime} \mathrm{N} 36^{\circ} 42^{\prime} 40.4^{\prime \prime} \mathrm{E}, 63^{\circ} 19^{\prime} 33.4^{\prime \prime} \mathrm{N}$ 36²'40.4”E, AbVi, Basp, Psph, 01-08.07.2016, leg. TP, SYKO.

Athallia PYRACEA (Ach.) Arup et al. - on bark of

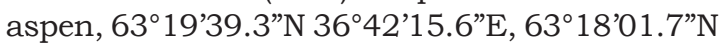
3641'12.9”'E, AbVi, Aher, 16.06.2011, leg. VT, PZV.

! BACIDINA ASSUlata (Körb.) S. Ekman - on bark of willow, $63^{\circ} 18^{\prime} 01.7^{\prime \prime} \mathrm{N} 36^{\circ} 41^{\prime} 12.9^{\prime \prime} \mathrm{E}$, Aher, 16.06.2011, leg. VT, PZV. Nemoral species with the Euroasian and American distribution range (Fadeeva, 2005). Distribution in Russia: Republic of Karelia, Leningrad Region, Kaliningrad Region, Omsk Region, Novosibirsk Region, Eastern Sayan Mountains, Primorsky Region (Golubkova, 2003). The closest finding: province of Karelia onegensis in the Republic of Karelia (Fadeeva et al., 2007). Distribution in the world: Europe, Asia, North America (Golubkova, 2003).

Biatora Beckhausi (Körb.) Tuck. - on bark of willow, $63^{\circ} 18^{\prime} 01.7^{\prime \prime} \mathrm{N} 36^{\circ} 41^{\prime} 12.9^{\prime \prime} \mathrm{E}$, Aher, 16.06.2011; on bark of birch and rowan, 6330'51.1"N 36³7'28.2"E, Sbir, Sroc, 08.06.2018, leg. VT, PZV. 
Biatora Helvola Körb ex Hellb. - on bark of

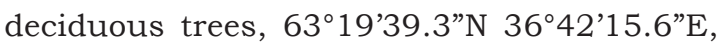
633'51.1"N 36³7'28.2”E, Sbir, Sroc, AbVi, 16.06.2011, 08.06.2018, leg. VT, PZV.

! Biatora HeMipolia (Nyl.) S. Ekman \& Printzen on bark of willow, 6319'39.3"N 36 42'15.6”"E, Rsh, 16.06.2011, leg. VT, PZV.

! Biatora Pallens (Kullh.) Printzen - on trunk and branches of juniper, on decaying wood, 6330'51.1"N 36³7'28.2"E, Sbir, Sroc,

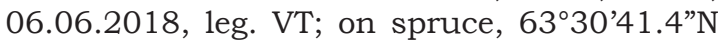

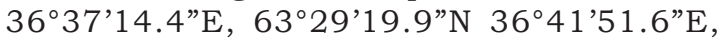
Ssph, Sher, 14.06.17, 23.06.2018, leg. AV, PZV; on bark of willow, on branches of spruce and

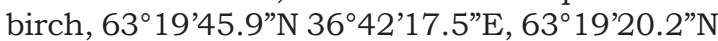
36²'57.1"E, Pmyr, Bher, 25.06.-01.07.2016, leg. ECh, TP, det. TP, SYKO.

! Bilimbia microcarpa (Th. Fr.) Th. Fr. - on bark of aspen, $63^{\circ} 20^{\prime} 53.6^{\prime \prime} \mathrm{N} 36^{\circ} 33^{\prime} 18.5^{\prime \prime} \mathrm{E}$, Smyr, 14.06.2011, leg. VT; 6330'25.2"N 36³6'54.7"E, Sher, 6330'8"N 36³8'20.5”E, Smyr, 13.06.2017, 24.06.2018, leg. AV, PZV.

Bilimbia sabuletorum (Schreb.) Arnold - on bark and mossy trunk of aspen, $63^{\circ} 17^{\prime} 58.4^{\prime \prime} \mathrm{N}$

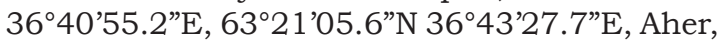
Basp, 06.07.2016, leg. TP, SYKO.

BRYORIA BICOLOR (Ehrh.) Brodo \& D. Hawksw - on birch, 63³0'51.1”N 36³7'28.2”E, Sbir, Sroc, Ssph, 11.06.2017, leg. AV; on boulders, 06.06.2018, leg. VT, PZV.

BRYORIA NADVORNIKIANA (Gyeln.) Brodo \& D. Hawksw. - on bark and wood of spruce and birch, juniper, rowan, pine, $63^{\circ} 23^{\prime} 47.1^{\prime \prime} \mathrm{N} 36^{\circ} 36^{\prime} 43.1^{\prime \prime} \mathrm{E}$, $63^{\circ} 22^{\prime} 36.7^{\prime \prime N} 36^{\circ} 46^{\prime} 36.4^{\prime \prime} \mathrm{E}, 63^{\circ} 30^{\prime} 51.1^{\prime \prime} \mathrm{N}$

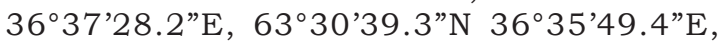
Ssph, Sher, Sroc, Sbir, 18.06.2010, 15.06.2011, 11.06.2018, leg. VT; 11.06.2017, leg. AV, PZV; $63^{\circ} 19^{\prime} 20.2^{\prime \prime N} 36^{\circ} 42^{\prime} 57.1$ "E, 63 $20^{\circ} 01.9^{\prime \prime} \mathrm{N}$ $36^{\circ} 42-37.8^{\prime \prime} \mathrm{E}, 63^{\circ} 19^{\prime} 19.6^{\prime \prime} \mathrm{N} 36^{\circ} 43^{\prime} 12.2^{\prime \prime} \mathrm{E}$, $63^{\circ} 20^{\prime 2} 4.3^{\prime \prime N} 36^{\circ} 43^{\prime 2} 22.9^{\prime \prime} \mathrm{E}$, Bher, Bsph, Spin, 01-06.07.2016, leg. TP, SYKO.

BRYoRia Simplicior (Vain.) Brodo \& D. Hawksw. - on bark and wood of pine and spruce,

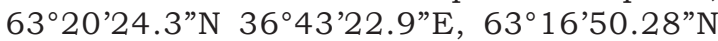
36³9'35.28”E, Bsph, Pmyr, 05-07.06.2016, leg. TP, SYKO.

BUELLIA GRISEOVIRENS (Turner \& Borrer ex Sm.)

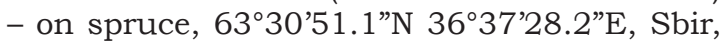
Sroc, on decaying wood, 06.06.2018, leg. VT; 63⒉'19.9”N 3641'51.6”'E, Sher, 14.06.2017, leg. AV, PZV; on bark of trunks and stumps of birch, 6320'01.9” ${ }^{\circ} 36^{\circ} 42^{\prime} 37.8^{\prime \prime} \mathrm{E}, 63^{\circ} 20^{\prime} 07.2^{\prime \prime} \mathrm{N}$ $36^{\circ} 40^{\prime} 06.4$ "E, Bsph, Bher, 01-03.07.2016, leg. TP, SYKO.

Calicium salicinum Pers. - on wood of spruce, $63^{\circ} 21^{\prime} 46.7^{\prime \prime N} 36^{\circ} 44^{\prime} 00.1^{\prime \prime} E$, Sher, 05.06.2016, leg. TP, SYKO.

Caloplaca BOREalis (Vain.) Poelt - on smooth bark of rowan, $63^{\circ} 30^{\prime} 51.1^{\prime \prime} \mathrm{N} 36^{\circ} 37^{\prime 2} 28.2^{\prime \prime E}$, Sbir, Sroc, 06.06.2018, leg. VT, PZV.

! Caloplaca sorocarpa (Vain.) Zahlbr. - on

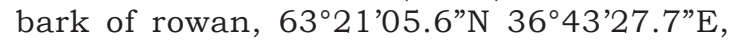

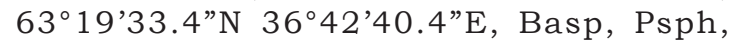
02.07.2016, 08.07.2016, leg. TP, SYKO.

Candelariella vitellina (Hoffm.) Müll. Arg. - on bark of willow, $63^{\circ} 19^{\prime} 39.3^{\prime \prime} \mathrm{N} 36^{\circ} 42^{\prime} 15.6$ " E, AbVi, Rsh, 16.06.2011, leg. VT, PZV.

! Candelariella xanthostigma (Ach.) Lettau on bark of willow and aspen, $63^{\circ} 19^{\prime} 39.3^{\prime \prime} \mathrm{N}$

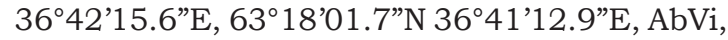
Rsh, Aher, 16.06.2014, leg. VT; on spruce, 6329'26.3"N 36³4'9.1"E, Sher, 12.06.2017, leg. AV, PZV; on worked timber and bark of rowan, 63\%19'45.9” N 3642'17.5” E, AvVi, 01.06.2016, leg. TP, SYKO.

! Carbonicola myrmecina (Ach.) Bendiksby \& Timdal - on burnt pine wood, $63^{\circ} 17^{\prime} 37.6^{\prime \prime} \mathrm{N}$ 3640’52.1”E, Psph, 06.07.2016, leg. TP, SYKO.

! CATILlaria ERYsiboides (Nyl.) Th. Fr. - on branch

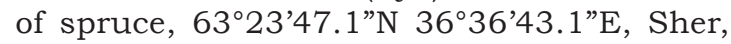
15.06.2014, leg. VT, PZV.

CAtinaria atropurpurea (Schaer.) Vězda \& Poelt - on bark of willow and aspen, birch,

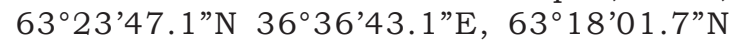
$36^{\circ} 41^{\prime} 12.9^{\prime \prime} \mathrm{E}, 6^{\circ} 30^{\prime} 51.1^{\prime \prime N} 36^{\circ} 37^{\prime} 28.2^{\prime \prime} \mathrm{E}$, Sher, Aher, Sbir, Sroc, 15-16.06.2014, 08.06.2018,

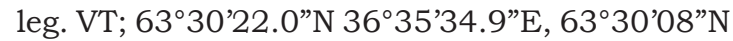
36 38'20.5” E, Psph, Smyr, 12.06.2017, 24.06.2018, leg. AV, PZV.

! CATINARIA NEUSCHILDII (Körb.) P. James - on bark of aspen, $63^{\circ} 20^{\prime} 53.6^{\prime \prime N} 36^{\circ} 33^{\prime} 18.5^{\prime \prime} \mathrm{E}$, Sbir, Sroc, 06.06.2018, leg. VA; 6330'25.2”"N 36³6'54.7”E, Sher, 13.06.2017, leg. AV, PZV.

Cetraria odontella (Ach.) Ach. - on stones and boulders with thin soil layer, 6330'51.1"N 36³7'28.2”E, Sbir, Sroc, 09.06.2018, leg. VT, PZV.

Chaenotheca BRachypoda (Ach.) Tibell - on wood of aspen and spruce, $63^{\circ} 20^{\prime} 53.6^{\prime \prime} \mathrm{N} 36^{\circ} 33^{\prime} 18.5^{\prime \prime} \mathrm{E}$, 
6330'51.1"N 36³7’28.2”E, Smyr, Sbir, Sroc, 14.06.2011, 09.06.2018, leg. VT, PZV; on wood of fallen tree and stumps of birch, $63^{\circ} 21^{\prime} 02.1^{\prime \prime} \mathrm{N}$ 3643'30.7"E, 6317'58.4"N 3640'55.2”E, Spin, Aher, 03.07.2016, leg. TP, SYKO.

! Chaenotheca laevigata Nádv. - on wood of birch stump, 6319'33.9”N 36³9'10.0”E, Bher, 03.07.2016, leg. TP, SYKO.

! Chaenotheca Phaeocephala (Turner) Th. Fr. - on bark of spruce, $63^{\circ} 20^{\prime} 53.6^{\prime \prime} \mathrm{N} 36^{\circ} 33^{\prime} 18.5^{\prime \prime} \mathrm{E}$, Sbir, Sroc, 08.06.2018, leg. VT, PZV; on bark of pine, 6319’33.9”N 36³9'10.0”E, Bher, 03.07.2016, leg. TP, SYKO.

Chaenotheca stemonea (Ach.) Müll. Arg. - on bark of spruce, $63^{\circ} 23^{\prime} 47.1^{\prime \prime N} 36^{\circ} 36^{\prime} 43.1^{\prime \prime} \mathrm{E}$, Sher, Smyr, 15.06.2011, leg. VT, PZV; 6320'38.4”N

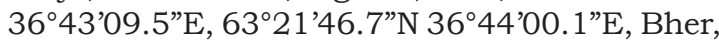
Sher, 04.07.2016, leg. TP, SYKO.

! \#ChAENothecopsis nigra Tibell - on thalli of Chaenotheca stemonea and C. furfuracea growing on bark and roots of spruce, 63 $20^{\circ} 53.6^{\prime \prime} \mathrm{N}$

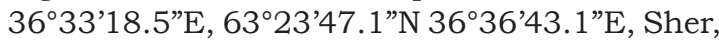
Smyr, 14-15.06.2011, leg. VT, PZV.

! \#Chaenothecopsis Pusilla (Ach.) A. F. W. Schmidt - on thalli of Chaenotheca sp. and free-living algae over bark and wood of spruce, 63 $30^{\prime} 51.1^{\prime \prime} \mathrm{N}$ 36³7'28.2"E, Sbir, Sroc, 08.06.2018, leg. VT; 6334'05.2”N 36²8'11.2”E, Smyr, Ssph,

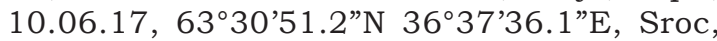
24.06.2018, leg. AV, PZV; 63⒉1'17.2”N

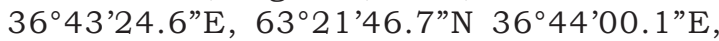
$63^{\circ} 17^{\prime 2} 22.9^{\prime \prime N} 36^{\circ} 40^{\prime 2} 24.1$ 'E, Psph, Sher, Pspr, 01-03.07.2016, leg. TP, SYKO.

! CHEIROMYCINA FLABELLIFORMis B. Sutton - on bark of willow, $63^{\circ} 21^{\prime} 05.6^{\prime \prime} \mathrm{N} 36^{\circ} 43^{\prime} 27.7^{\prime \prime} \mathrm{E}$, Basp, 02.07.2016, leg. TP, SYKO.

! CHRYSOTHRIX CANDELARIS (L.) J. R. Laundon - on shaded rock, 63²0'53.6”N 36³3'18.5”E, Smyr, 09.06.2018, leg. VT, PZV.

CHRYSOTHRIX CHLORINA (Ach.) J. R. Laundon on shaded rock, on boulders, $63^{\circ} 17^{\prime} 58.4^{\prime \prime} \mathrm{N}$ 3640'55.2”E, 6316'15.06”N 36³9'49.26”E, R, Pmyr, 02-07.07.2016, leg. TP, SYKO.

Cladonia Bellidiflora (Ach.) Schaer. - on stones and boulders with thin soil layer and on mossy boulders, 6330'51.1"N 36³7'28.2” E, Sbir, Sroc, 09.06.2018, leg. VT; 63³4'05.2”N 36²8'11.2"E, Sbir, Sroc, 11-15.06.17, leg. AV, $\mathrm{PZV}$; 6320'27.12”N 36³6’09.78”E, 6317'09.3”N
36³9'34.7”E, Sroc, Pspr, 03.06.2016, leg. TP, SYKO. RA.

Cladonia carneola (Fr.) Fr. - on decaying wood, 6330'51.1"N 36³7'28.2”E, Sbir, Sroc, 09.06.2018, leg. VT, PZV.

Cladonia chlorophaea (Flörke ex Sommerf.) Spreng. - on soil, at trunk base, on fallen trees, 6330'51.1"N 36³7'28.2"E, Sbir, Sroc, Smyr, 09.06.2018, leg. VT; on stones, 1011.06.2017, leg. AV, PZV; on birch and boul-

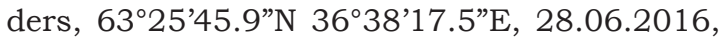
$63^{\circ} 20^{\prime 2} 2.12$ "N 36³6'09.78”E, Sher, Sroc, 28.06-03.07.2016, leg. ECh; on soil and bark of willow, 6319'45.9” $\mathrm{N} 36^{\circ} 42^{\prime} 17.5^{\prime \prime} \mathrm{E}, 63^{\circ} 20^{\prime} 01.9^{\prime \prime} \mathrm{N}$

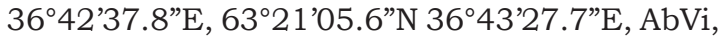
Bsph, Basp, 03.07.216, leg. TP, SYKO. The specimens contain fumarprotocetraric acid.

Cladonia cornuta subsp. GRoenlandica (Å.E. Dahl) Ahti - on stones and boulders with thin soil layer, 6330'51.1"N 36³7'28.2"E, Sbir, Sroc, 08.06.2018, leg. VT \& AV; 63³4’05.2"N 36²8'11.2”E, 6329'19.9”N 3641'51.6”'E, Sbir, Sroc, Sher, 12-14.06.2017, leg. AV, PZV.

Cladonia CYANiPES (Sommerf.) Nyl. - on stones and boulders with thin soil layer, 6330'51.1"N 36³7'28.2”'E, Sbir, Sroc, 09.06.2018, leg. VT, PZV.

Cladonia macrophylla (Schaer.) Stenh. - on stones and boulders with thin soil layer, on mossy boulders, 6330'51.1"N 36³7'28.2” E, Sbir, Sroc, 09.06.2018, leg. VT; 11.06.2017, leg. AV, PZV; 6316'15.06”N 36³9'49.26”E, R, 03.06.2016, leg. ECh, det. TP, SYKO.

! Cladonia novochlorophaea (Sipman) Brodo $\&$ Ahti - on worked timber, 63 $19^{\prime} 45.9^{\prime \prime} \mathrm{N}$ 36²'17.5”E, 01.07.2016, leg. TP, det. I. S. Stepanchikova, SYKO. The specimens contain fumarprotocetraric, homosekikaic and sekikaic acids.

Cladonia OCHROCHLORA Flörke - on stones and boulders with thin soil layer, 6330'51.1"N 36³7'28.2”'E, Sbir, Sroc, 06.06.2018, leg. VT, PZV.

Cladonia Phyllophora Hoffm. - on stones and boulders with thin soil layer, 6330'51.1"N 36³7'28.2"E, Sbir, Sroc, 06.06.2018, leg. VT; 11.06.2017, leg. AV, PZV; 63 $20^{\prime} 40.3^{\prime \prime} \mathrm{N}$ $36^{\circ} 43^{\prime} 24.6^{\prime \prime} \mathrm{E}, 63^{\circ} 21^{\prime} 02.0^{\prime \prime} \mathrm{N} 36^{\circ} 43^{\prime} 30.0^{\prime \prime} \mathrm{E}$, $63^{\circ} 17^{\prime} 18.6^{\prime \prime N ~} 36^{\circ} 39^{\prime} 50.4^{\prime} \mathrm{E}, \mathrm{FR}$, Proc, 03.06.2016, leg. TP, SYKO. 
Cladonia polydactyla (Flörke) Spreng. - on boulders with thin soil layer and trunk base of spruce, $63^{\circ} 25^{\prime} 45.9^{\prime \prime} \mathrm{N} 36^{\circ} 38^{\prime} 17.5^{\prime \prime} \mathrm{E}$, Sher, 28.06.2016, leg. ECh; 6320'40.3"N 3643'24.6” E, FR, 6319'19.6” $36^{\circ} 43^{\prime} 12.2^{\prime \prime} \mathrm{E}$, Psph, 02-03.07.2016, leg. TP, SYKO.

Cladonia subfurcata (Nyl.) Arnold - on stones and boulders with thin soil layer, on mossy boulders, 6330'51.1"N 36³7'28.2"E, Sbir, Sroc, 06.06.2018, leg. VT, PZV; 63 $25^{\prime} 14.76$ "N $36^{\circ} 38^{\prime} 13.62 " \mathrm{E}, 63^{\circ} 16^{\prime} 15.06^{\prime \prime} \mathrm{N} 36^{\circ} 39^{\prime} 49.26^{\prime \prime} \mathrm{E}$, Sw, Plich, R, 26.06-3.07.2016, leg. ECh, det. TP, SYKO.

Cladonia verticillata (Hoffm.) Schaer. - on mossy boulder, 6317'18.6”N 36³9'50.4"E, Proc, 06.07.2016, leg. TP, SYKO.

Coenogonium Pineti (Ach.) Lücking \& Lumbsch - on smooth bark of rowan, 63 $30^{\prime} 51.1^{\prime \prime} \mathrm{N}$ 36³7'28.2"E, Sbir, Sroc, 06.06.2018, leg. VT;

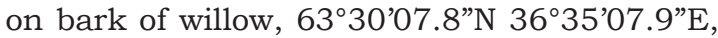
Sbir, 06.06.2018, leg. AV, PZV.

COLlema FURFURACEUm (Arnold) Du Rietz - on bark of aspen, $63^{\circ} 23^{\prime} 47.1^{\prime \prime N} 36^{\circ} 36^{\prime} 43.1^{\prime \prime} E$, Sher, 15.06.2011, leg. VT, PZV.

Diplotomma alboatrum (Hoffm.) Flot. - on bark of willow, 6319'39.3”N 3642'15.6”E, Rsh, 16.06.2011, leg. VT, PZV

! EPHEBE LANATA (L.) Vain. - on rocks, $63^{\circ} 16^{\prime} 15.06^{\prime \prime N}$ 36³9'49.26”E, R, 07.07.2016, leg. ECh, det. TP, SYKO.

EVERNIA PRUNASTRI (L.) Ach. - on worked timber, on bark of willow, rowan and pine, rarely

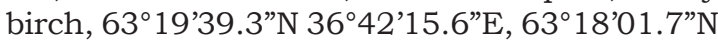
$36^{\circ} 41^{\prime} 12.9^{\prime \prime} \mathrm{E}, \mathrm{AbVi}$, Aher, 16.06.2011, leg. VT; 6330'07.8” N 36 35'07.9” E, Psph, 06.06.2018, leg. AV, PZV; 6320'01.9”N 36 42'37.8”, $63^{\circ} 20^{\prime} 38.4^{\prime \prime} \mathrm{N} 36^{\circ} 43^{\prime} 09.5^{\prime \prime} \mathrm{E}, 63^{\circ} 21^{\prime} 05.6^{\prime \prime} \mathrm{N}$ 3643’27.7”E, Bsph, Basp, 02.07.2016, leg. TP, SYKO.

! EOPYRENULA LEUCOPLACA (Wallr.) R.C. Harris - on bark of aspen, $63^{\circ} 17^{\prime} 22.9^{\prime \prime N} 36^{\circ} 40^{\prime} 24.1^{\prime \prime} \mathrm{E}$, Ssph, 03.07.2016, leg. TP, SYKO.

FlaVocetraria NIVALIS (L.) Kärnefelt \& A. Thell - on stones and boulders with thin soil layer, 6330'51.1"N 36³7'28.2"E, Sbir, Sroc, 09.06.2018, leg. VT, PZV.

! FRUTIDELLA CAESIOATRA (Schaer.) Kalb - on stones and boulders with thin soil layer, 63 30 '51.1"N 36³7’28.2”E, Sbir, Sroc, 11.06.2018, leg. VT, PZV.
! Gyalecta fagicola (Hepp ex Arnold) Kremp. - on bark of willow, aspen and birch, $63^{\circ} 18^{\prime} 01.7^{\prime \prime} \mathrm{N}$ $36^{\circ} 41^{\prime} 12.9^{\prime \prime} \mathrm{E}$, Aher, 16.06.2011, leg. VT, PZV; $63^{\circ} 20^{\prime} 01.9^{\prime \prime} \mathrm{N} \mathrm{36^{ \circ } 4 2} 37.8^{\prime \prime} \mathrm{E}, 63^{\circ} 21^{\prime} \mathrm{O} 2.1^{\prime \prime} \mathrm{N}$ 3643'30.7”E, Bsph, Psb, 02-05.07.2016, leg. TP, SYKO.

! Gyalecta truncigena (Ach.) Hepp - on smooth bark of young aspen, $63^{\circ} 20^{\prime} 53.6^{\prime \prime} \mathrm{N} 36^{\circ} 33^{\prime} 18.5^{\prime \prime} \mathrm{E}$, Sbir, Sroc, 09.06.2018, leg. VT, PZV.

Gyalolechia flavorubescens (Huds.) Søchting et al. - on bark of aspen, $63^{\circ} 20^{\prime} 53.6^{\prime \prime} \mathrm{N}$ $36^{\circ} 33^{\prime} 18.5^{\prime \prime} \mathrm{E}, 63^{\circ} 19^{\prime} 54.5^{\prime \prime} \mathrm{N} 36^{\circ} 34^{\prime} 49.0^{\prime \prime} \mathrm{E}$,

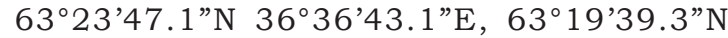

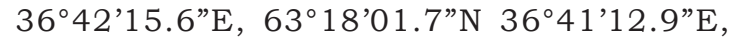
Smyr, Proc, Sher, AbVi, Aher, 11-16.06.2018, leg. VT, PZV.

Hypogymia BitTeri (Lynge) Ahti - on bark of spruce, birch and wood, 6320'53.6” N 36³3'18.5”E, Sbir, Sroc, 09.06.2018, leg. VT, PZV.

LECANia CYRTELLA (Ach.) Th. Fr. - on smooth bark of willow, aspen and rowan, $63^{\circ} 23^{\prime} 47.1^{\prime \prime} \mathrm{N}$

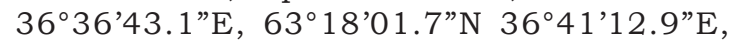
$63^{\circ} 30^{\prime} 51.1^{\prime \prime} \mathrm{N} 36^{\circ} 37^{\prime} 28.2$ " E, Sher, Aher, Sbir, Sroc, 15-16.06.2011, 09.06.2018, leg. VT, PZV.

! LeCAnia Cyrtellina (Nyl.) Sandst. - on bark of willow, aspen and pine, 63 $20^{\prime} 53.6^{\prime \prime} \mathrm{N}$ $36^{\circ} 33^{\prime} 18.5^{\prime \prime} \mathrm{E}, 63^{\circ} 19^{\prime} 54.5^{\prime \prime} \mathrm{N} 36^{\circ} 34^{\prime} 49.0^{\prime \prime} \mathrm{E}$, $63^{\circ} 18^{\prime} 01.7^{\prime \prime} \mathrm{N} 36^{\circ} 41^{\prime} 12.9^{\prime \prime} \mathrm{E}, 63^{\circ} 30^{\prime} 51.1^{\prime \prime} \mathrm{N}$ $36^{\circ} 37^{\prime 2} 2.2^{\prime \prime} \mathrm{E}$, Smyr, Proc, Aher, Sbir, Sroc, 14-16.06.2011, 09.06.2018, leg. VT, PZV.

LeCANiA NAEGELII (Hepp) Diederich \& van den Boom - on bark of willow, aspen and pine, 6320'53.6” N 36³3'18.5” E, 6319'39.3” N 36²'15.6"E, Smyr, AbVi, 14-16.06.2011, leg. VT, PZV.

! LECANORA AITEMA (Ach.) Hepp - on thin branches of spruce, $63^{\circ} 19^{\prime} 33.9^{\prime \prime} \mathrm{N} 36^{\circ} 39^{\prime} 10.0 " \mathrm{E}$, Bher, 03.07.2016, leg. TP, SYKO.

LeCANORA AlBellula var. Albellula (Nyl.) Th. Fr. - on bark of aspen and juniper, $63^{\circ} 23^{\prime} 47.1^{\prime \prime} \mathrm{N}$ 36³6'43.1"E, 6330'51.1"N 36³7'28.2"E, Sher, Sbir, Sroc, 16.06.2011, 09.06.2018, leg. VT; on trunk and branches of birch, 63 $30^{\prime} 52.2^{\prime \prime} \mathrm{N}$ 36³4'46.4”E, Sbir, 23.06.2018, leg. AV, PZV.

LECANORA ALLOPHANA Nyl. - on bark of willow, aspen and rowan, 6320'53.6” $\mathrm{N} 36^{\circ} 33^{\prime} 18.5^{\prime \prime} \mathrm{E}$,

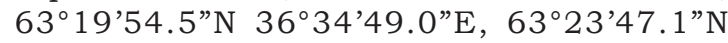


$36^{\circ} 36^{\prime} 43.1^{\prime \prime} \mathrm{E}, 63^{\circ} 19^{\prime} 39.3^{\prime \prime} \mathrm{N} 36^{\circ} 42^{\prime} 15.6^{\prime \prime} \mathrm{E}$, $63^{\circ} 18^{\prime} 01.7^{\prime \prime} \mathrm{N} 36^{\circ} 41^{\prime} 12.9^{\prime \prime} \mathrm{E}, 63^{\circ} 30^{\prime} 51.1^{\prime \prime} \mathrm{N}$ 36 37'28.2"E, Smyr, Proc, Sher, AbVi, Aher, Sbir, Sroc, 14-16.06.2011, 09.06.2018, leg. VT; 6330'25.2”N 36³6’54.7”E, 63²9'55.1”N

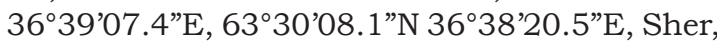
Smyr, 13.06.2017, 24.06.2018, leg. AV, PZV; $63^{\circ} 25^{\prime} 45.9^{\prime \prime} \mathrm{N} 36^{\circ} 38^{\prime} 17.5^{\prime \prime} \mathrm{E}$, Pmyr, 27.06.2016, leg. ECh, $63^{\circ} 19^{\prime} 33.4^{\prime \prime N} 36^{\circ} 42^{\prime} 40.4^{\prime \prime} \mathrm{E}, \mathrm{Bsph}$, 03.07.2016, leg. TP, SYKO.

! LECANORA ARGENTATA (Ach.) Malme - on worked timber, 6319'39.3” N 3642'15.6” E, AbVi, 16.06.2011, leg. VT, PZV.

! LECANORA CARPINEA (L.) Vain. - on bark of aspen and rowan, 63 $20^{\prime} 53.6^{\prime \prime} \mathrm{N} 36^{\circ} 33^{\prime} 18.5^{\prime \prime} \mathrm{E}$, $63^{\circ} 19^{\prime} 39.3$ "N 3642'15.6” E, 6318'01.7”N 36 41'12.9"E, Smyr, AbVi, Aher, 16.06.2011, leg. VT, PZV; 63⒉ $5^{\prime} 45.9^{\prime \prime} \mathrm{N} 36^{\circ} 38^{\prime} 17.5^{\prime \prime} \mathrm{E}$,

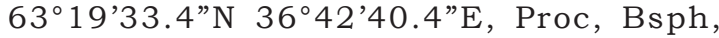
07.07.2016, leg. TP, SYKO.

Lecanora Cateilea (Ach.) A. Massal. - on smooth bark of young rowan, 6330'51.1"N 36³7'28.2”E, Sbir, Sroc, 11.06.2018, leg. VT, PZV.

LECANORA CIRCUMBOREALIS Brodo \& Vitik. - on

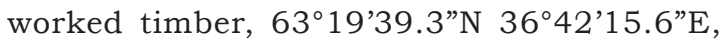
$63^{\circ} 30^{\prime} 51.1^{\prime \prime} \mathrm{N} 36^{\circ} 37^{\prime} 28.2$ "E, AbVi, 16.06.2011, Sbir, Sroc, on bark of rowan, 09.06.2018, leg. VT, PZV.

LECANORA INTRICATA (Ach.) Ach. - on stones and boulders, 6330'51.1"N 36³7’28.2”E, Sbir, Sroc, 11.06.2018, leg. AS, PZV.

! LeCANora saligna (Schrad.) Zahlbr. - on worked timber, 6319'39.3"N 3642'15.6” $\mathrm{E}$, AbVi, 16.06.2011, leg. VT, PZV; 6319'45.9”N 36²'17.5”E, AbVi, 03.07.2016, leg. TP, SYKO.

! LECANORA CF. SUBINTRICATA (Nyl.) Th. Fr. - on bark and wood of pine, $63^{\circ} 17^{\prime} 37.6^{\prime \prime} \mathrm{N} 36^{\circ} 40^{\prime} 52.1^{\prime \prime} \mathrm{E}$, 6317'58.4”N 3640'55.2"E, Psph, Pmyr, 03.07.2016, leg. TP, SYKO.

! LeCANORA VARIa (Hoffm.) Ach. - on worked timber, 6319'45.9” N 36 42'17.5” E, AbVi, 04.07.2016, leg. TP, SYKO.

LECIDEA ERYTHROPHAEA Flörke ex Sommerf. - on

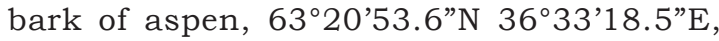
Smyr, 14.06.2011, leg. VT, PZV; 63¹9'33.4”N $36^{\circ} 42^{\prime} 40.4^{\prime \prime} \mathrm{E}, 63^{\circ} 21^{\prime} 05.6^{\prime \prime} \mathrm{N} 36^{\circ} 43^{\prime} 27.7^{\prime \prime} \mathrm{E}$, $63^{\circ} 17^{\prime} 58.4^{\prime \prime} \mathrm{N} 36^{\circ} 40^{\prime} 55.2$ "E, Basp, Aher, 0105.07.2016, leg. TP, SYKO.
LECIDEA tuRgidula Fr. - on bark, wood and branches of spruce, on pine, $63^{\circ} 22^{\prime} 36.7^{\prime \prime} \mathrm{N}$ 364'36.4”E, Ssph, Smyrt, 16.06.2011, leg. VT; $63^{\circ} 30^{\prime} 51.1^{\prime \prime N} 36^{\circ} 37$ '28.2” E, Ssph, on branches of spruce, 11.06.2017, leg. AV, PZV; 6320'07.2”N 36³9'58.4"E, Psph, 06.07.2016, leg. TP, SYKO.

Lecidella elaeochroma (Ach.) M. Choisy - on bark of aspen, $63^{\circ} 17^{\prime} 58.4^{\prime \prime} \mathrm{N} 36^{\circ} 40^{\prime} 55.2^{\prime \prime} \mathrm{E}$, Aher, 06.07.2016, leg. TP, SYKO.

LEPTOGIUM SATURNINUM (Dicks.) Nyl. - on bark of

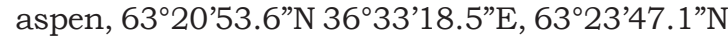
$36^{\circ} 36^{\prime} 43.1^{\prime \prime} \mathrm{E}, 63^{\circ} 18^{\prime} 01.7^{\prime \prime} \mathrm{N} 36^{\circ} 41^{\prime} 12.9^{\prime \prime} \mathrm{E}$, Smyr, Sher, Aher, 14-16.06.2011, leg. VT, PZV; $63^{\circ} 17^{\prime} 58.4^{\prime \prime N} 36^{\circ} 40$ '55.2”E, Aher, 05.07.2016, leg. TP, SYKO.

! LePraria membranacea (Dicks.) Vain. - on

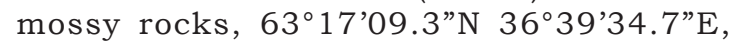
6316'15.06” N 36³9'49.26" E, Proc, R, 06.07.2016, leg. ECh \& TP, det. TP, SYKO.

! Micarea Globulosella (Nyl.) Coppins - on bark of spruce, birch, aspen, on standing deadwood, 6330'51.1"N 36³7’28.2”E, Sbir, Sroc, 06.06.2018, leg. VT, PZV.

! Micarea peliocarpa (Anzi) Coppins \& R. Sant. - on fallen spruce tree, 6330'51.1"N 36³7'28.2”E, Sbir, Sroc, 06.06.2018, leg. VT, PZV.

! Montanelia PANNIFORMIS (Nyl.) Divakar et al.- on stones and boulders, 63⒊'14.4"N 36 61'40.2” E Proc, R, 13.06.2011, leg. AS, PZV.

Mycobilimbia EPIXANTHOIDES (Nyl.) Vitik. et al. on bark of aspen, $63^{\circ} 20^{\prime} 53.6^{\prime \prime} \mathrm{N} 36^{\circ} 33^{\prime} 18.5^{\prime \prime} \mathrm{E}$,

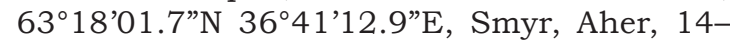
16.06.2011, leg. VT; 6330’39.9”N 36³7’20.9”E, $63^{\circ} 30^{\prime} 08^{\prime \prime N} 36^{\circ} 38^{\prime 2} 20.5$ ”E, Smyr, 23-24.06.2018, leg. AV, PZV.

! Mycobilimbia tetramera (De Not.) Vitik. et al. - on mosses and bark of aspen, 6317'58.4"N 36 40'55.2"E, Aher, 06.07.2016, leg. TP, SYKO; 633’51.1”N 36³7’28.2”E, Sbir, Sroc, 11.06.2018, leg. VT, PZV.

Myriolecis hageni (Ach.) Śliwa et al - on worked timber, 6319'45.9” N 3642'17.5” E, AbVi, 01-04.07.2016, leg. TP, SYKO; 6330'51.1”N $36^{\circ} 37^{\prime 2} 2.2^{\prime \prime} \mathrm{E}$, Sbir, Sroc, on bark of rowan and alder, 09-11.06.2018, leg. VT, PZV.

! Myriolecis sambuci (Pers.) Clem. $-63^{\circ} 19 ’ 39.3^{\prime \prime N}$ $36^{\circ} 42^{\prime} 15.6^{\prime \prime} \mathrm{E}, \mathrm{AbVi}$, on bark of willow, aspen and worked timber, 16.06.2011, leg. VT; 
6329'55.1"N 36³9'07.4"E, Smyr, on bark of aspen, 23.06.2018, leg. AV, PZV.

! Naetrocymbe punctiformis (Pers.) R.C. Harris - on smooth bark of willow and rowan, 6330'51.1"N 36 37'28.2"E, Sbir, Sroc, 07.06.2018, leg. VT, PZV.

NePhroma Bellum (Spreng.) Tuck. - on bark of willow and aspen, 63 $20^{\prime} 53.6^{\prime \prime} \mathrm{N} 36^{\circ} 33^{\prime} 18.5^{\prime \prime} \mathrm{E}$, $63^{\circ} 19^{\prime} 54.5^{\prime \prime} \mathrm{N} 36^{\circ} 34^{\prime} 49.0$ " E, 63 $18^{\circ} 01.7^{\prime \prime} \mathrm{N}$ $36^{\circ} 41^{\prime} 12.9^{\prime \prime} \mathrm{E}$, Smyr, Proc, Aher, 14-16.06.2011,

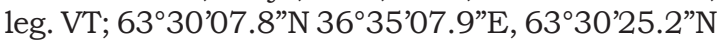
36³6'54.7'E, Smyr, 23.06.2018, leg. AV, PZV; $63^{\circ} 16^{\prime} 50.28^{\prime \prime} \mathrm{N} 36^{\circ} 39^{\prime} 35.28^{\prime} \mathrm{E}$, Proc, on bark

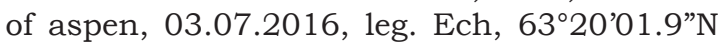

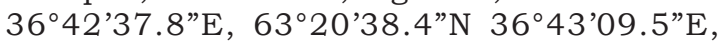
$63^{\circ} 21^{\prime} 05.6^{\prime \prime} \mathrm{N} 36^{\circ} 43^{\prime} 27.7^{\prime \prime} \mathrm{E}, 63^{\circ} 19^{\prime} 33.9^{\prime \prime} \mathrm{N}$ 36³9'10.0”E, 6320'24.3”N 3643'22.9”'E, Bsph, Bher, Basp, on bark of willow, birch, spruce, juniper, aspen, 01-03.07.2016, leg. TP, SYKO.

! OChrolechia BaHUSIEnsis H. Magn. - on bark of spruce and birch, 6332'11.2” N 36 35'12.8” E, Sroc, 11.06.2017, leg. AV, PZV; on bark of rowan, 63⒉ $1^{\prime} 05.6^{\prime \prime} \mathrm{N} 36^{\circ} 43^{\prime} 27.7^{\prime} \mathrm{E}$, Basp, 02.07.2016, leg. TP, det. I. S. Stepanchikova, SYKO. The specimens contain gyrophoric, lecanoric acids and murolic acid complex.

! Palicella filamentosa (Stirt.) Rodr. Flakus et Printzen - on bark of birch, $63^{\circ} 30^{\prime} 51.1^{\prime \prime} \mathrm{N}$ 36³7'28.2”E, Sbir, Sroc, 11.06.2018, leg. VT, PZV.

Parmeliella triptophylla (Ach.) Müll. Arg. - on bark of aspen, $63^{\circ} 23^{\prime} 47.1^{\prime \prime} \mathrm{N} 36^{\circ} 36^{\prime} 43.1^{\prime \prime} \mathrm{E}$, Sher, 15.06.2011, leg. VT; 6330'25.1”N 36³6'54.7”E, Smyr, 13.06.2017, leg. AV, PZV.

Peltigera Canina (L.) Willd. - on soil, mossy boulders, bark of aspen, $63^{\circ} 18^{\prime} 01.7^{\prime \prime} \mathrm{N} 36^{\circ} 41^{\prime} 12.9^{\prime \prime} \mathrm{E}$, Aher, 16.06.2011, leg. VT, PZV, confirm. O. Vitikainen; 6320'27.12”N 36³6'09.78” E, Sroc, on fallen tree, leg. ECh, $63^{\circ} 19^{\prime} 45.9^{\prime \prime} \mathrm{N} 36^{\circ} 42^{\prime} 17.5^{\prime \prime} \mathrm{E}$, $63^{\circ} 20^{\prime} 01.9^{\prime \prime} \mathrm{N} 36^{\circ} 42^{\prime} 37.8^{\prime \prime} \mathrm{E}, 63^{\circ} 21^{\prime} 05.6^{\prime \prime} \mathrm{N}$

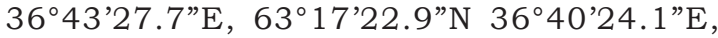
$63^{\circ} 17^{\prime} 58.4^{\prime \prime N} 36^{\circ} 40^{\prime} 55.2$ "E, AbVi, Rsh, Basp, Sroc, Psb, Aher, 01-03.07.2016, leg. TP, SYKO.

Peltigera didactyla (With.) J. R. Laundon on bark of willow and aspen, $63^{\circ} 23^{\prime} 47.1^{\prime \prime} \mathrm{N}$ 36³6'43.1"E, Sher, 16.06.2011, leg. VT, $\mathrm{PZV}$, confirm. O. Vitikainen; at trunk base of juniper and decaying wood, 63⒉ $20^{\prime} 24.3^{\prime \prime} \mathrm{N}$

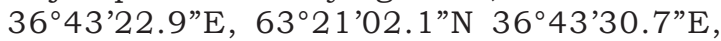
$63^{\circ} 21^{\prime} 02^{\prime \prime N ~} 36^{\circ} 43^{\prime} 30^{\prime} \mathrm{E}, \mathrm{Bsph}, \mathrm{Pspb}, \mathrm{FR}$, 03.07.2016, leg. TP, SYKO.
Peltigera malacea (Ach.) Funck - on soil, 6330'51.1"N 36³7'28.2"E, Sbir, Sroc, 10.06.2018, leg. VT, PZV, confirm. O. Vitikainen.

Peltigera polydactylon (Neck.) Hoffm. - on soil, 6330'51.1"N 36³7'28.2"E, Sbir, Sroc, 10.06.2018, leg. VT, PZV, confirm. O. Vitikainen; on falling trees, on mossy trunk base of trees, decaying wood, rock, $63^{\circ} 25^{\prime} 45.9^{\prime \prime} \mathrm{N}$ $36^{\circ} 38^{\prime} 17.5^{\prime \prime} \mathrm{E}, 63^{\circ} 20-27.12^{\prime \prime} \mathrm{N}, 36^{\circ} 36^{\prime} 09.78^{\prime \prime} \mathrm{E}$,

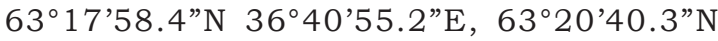
$36^{\circ} 43^{\prime} 24.6^{\prime \prime} \mathrm{E}, 63^{\circ} 25^{\prime} 45.9^{\prime \prime} \mathrm{N} 36^{\circ} 38^{\prime} 17.5^{\prime \prime} \mathrm{E}$,

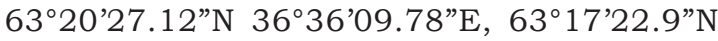
$36^{\circ} 40^{\prime 2} 4.1$ "E. Sher, Smyr, FR, Sher, Sroc, Pspr, Aher, 01-06.07.2016, leg. TP, SYKO.

! Peltigera ponojensis Gyeln. - on soil, $63^{\circ} 20^{\prime} 40.3$ "N 3643'24.6”E, FR, 02.07.2016, leg. TP, SYKO.

Peltigera praetextata (Flörke ex Sommerf.) Zopf - on bark of aspen, willow and birch, $63^{\circ} 19^{\prime} 54.5^{\prime} \mathrm{N} 36^{\circ} 34^{\prime} 49.0$ " E, Proc, 14.06.2011,

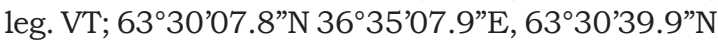
$36^{\circ} 37^{\prime 20.9 ”} \mathrm{E}, 63^{\circ} 30^{\prime} 08.0^{\prime \prime N} 36^{\circ} 38^{\prime 2} 20.5^{\prime \prime} \mathrm{E}$, Smyr, 12, 23-24.06.2018, leg. AV, PZV, confirm. O. Vitikainen; $63^{\circ} 20^{\prime} 01.9^{\prime \prime} \mathrm{N} 36^{\circ} 42^{\prime} 37.8^{\prime \prime} \mathrm{E}, \mathrm{Bsph}$, 01.07.2016, leg. TP, SYKO.

Peltigera Rufescens (Weiss) Humb. - on

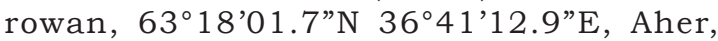
16.06.2011, leg. VT, PZV, confirm. O. Vitikainen; at trunk base of willow, on soil, $63^{\circ} 20^{\prime} 01.9^{\prime \prime} \mathrm{N}$ $36^{\circ} 42^{\prime} 37.8^{\prime \prime} \mathrm{E}, 63^{\circ} 19^{\prime} 45.9^{\prime \prime} \mathrm{N} 36^{\circ} 42^{\prime} 17.5^{\prime \prime} \mathrm{E}$, $63^{\circ} 21^{\prime} 02^{\prime \prime N} 36^{\circ} 43^{\prime} 30^{\prime \prime E}$, Bsph, AbVi, FR, 0105.07.2016, leg. TP, SYKO.

Peltigera scabrosa Th. Fr. - on stone with thin soil layer, 6319'54.5” N 36³4'49.0”E, Proc, 14.06.2011, leg. VT, PZV, confirm. O. Vitikainen; on soil, $63^{\circ} 21^{\prime} 02.0^{\prime \prime} \mathrm{N} 36^{\circ} 43^{\prime} 30.0$ ” E, FR, 05.07.2016, leg. TP, SYKO.

Pertusaria Carneopallida (Nyl.) Anzi ex Nyl. $63^{\circ} 30^{\prime} 51.1^{\prime \prime N} 36^{\circ} 37^{\prime} 28.2^{\prime \prime} \mathrm{E}$, Sbir, Sroc, on bark of rowan, 11.06.2018, leg. VT; 6332'11.2”N 36³5'12.8”'E, Ssph, on birch, 11.06.2017, leg. AV, PZV.

! Pertusaria leioplaca DC. - on bark of aspen, 6320'53.6”N 36³3'18.5”E, Smyr, 14.06.2011, leg. VT, PZV.

PeRTUSARIa OPHTHALMiza (Nyl.) Nyl. - on bark of birch, 6330'51.1"N 36³7'28.2"E, Sbir, Sroc, 11.06.2018, leg. VT; on trunk and branches of spruce, $63^{\circ} 30^{\prime} 41.4^{\prime \prime} \mathrm{N} 36^{\circ} 37^{\prime} 14.5^{\prime} \mathrm{E}$, Ssph, 15.06.2017, leg. AV, PZV. 
Pertusaria pupillaris (Nyl.) Th.Fr. - on bark of rowan, $63^{\circ} 21^{\prime} 0.1^{\prime \prime N} 36^{\circ} 43^{\prime} 30.7^{\prime \prime} \mathrm{E}, \mathrm{Psb}$, 05.07.2016, leg. TP, det. I. Stepanchikova, SYKO.

PhaEophyscia CiLIATA (Hoffm.) Moberg - on bark of aspen, 6320'53.6” $\mathrm{N} 36^{\circ} 33^{\prime} 18.5^{\prime \prime} \mathrm{E}, 63^{\circ} 19^{\prime} 39.3^{\prime \prime} \mathrm{N}$ 36²'15.6”'E, Smyr, AbVi, 14.06.2011, leg. VT,

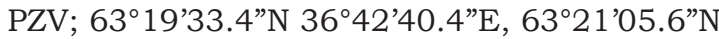
3643'27.7”E, Basp, 01.07.2016, leg. TP, SYKO.

Phlyctis ARgena (Spreng.) Flot. - on trunk and branches of spruce, 6330'51.1"N 36³7'28.2" E, Sbir, Sroc, on bark of willow, 11.06.2018, leg. VT; on bark of willow and aspen, 63 $30^{\prime} 41.1^{\prime \prime} \mathrm{N}$ $36^{\circ} 37^{\prime} 14.5^{\prime \prime} \mathrm{E}, 63^{\circ} 30^{\prime} 07.8^{\prime} \mathrm{N} 36^{\circ} 35^{\prime} 07.9^{\prime \prime} \mathrm{E}$, 6330'25.2”N 36 36'54.7"E, Ssph, Smyr, Sher, 12.06.2017, leg. AV, PZV; 63² $21^{\prime} 05.6^{\prime \prime} \mathrm{N}$ $36^{\circ} 43^{\prime} 27.7$ " E, Basp, on bark of aspen, 01.07.2016, leg. TP, SYKO.

Physcia Adscendens (Fr.) Oliv. - on bark of aspen, $63^{\circ} 18^{\prime} 01.7^{\prime \prime} \mathrm{N} 36^{\circ} 41^{\prime} 12.9$ 'E, Aher, 16.06.2011,

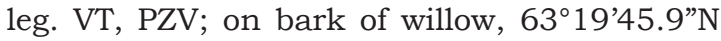
3642'17.5”'E, AbVi, 01.07.2016, leg. TP, SYKO.

Physcia aipolia (Ehrh. ex Humb.) Fürnr. on bark of aspen and willow, 63 $23^{\prime} 47.1^{\prime \prime} \mathrm{N}$ $36^{\circ} 36^{\prime} 43.1^{\prime \prime} \mathrm{E}, 63^{\circ} 18^{\prime} 01.7^{\prime \prime} \mathrm{N} 36^{\circ} 41^{\prime} 12.9^{\prime \prime} \mathrm{E}$, Sher, Aher, 15.06.2011, leg. VT, PZV; 6319'33.4"N $36^{\circ} 42^{\prime} 40.4^{\prime \prime} \mathrm{E}, 63^{\circ} 21^{\prime} 05.6^{\prime \prime} \mathrm{N} 36^{\circ} 43^{\prime} 27.7^{\prime \prime} \mathrm{E}$, $63^{\circ} 17^{\prime} 58.4^{\prime \prime} \mathrm{N} 36^{\circ} 40^{\prime} 55.2$ "E, Basp, Aher, 0105.07.2016, leg. TP, SYKO.

! Physcia ALnophila (Vain.) Loht. et al. - on bark of rowan, aspen and willow, 63 $20^{\prime} 53.6^{\prime \prime} \mathrm{N}$ $36^{\circ} 33^{\prime} 18.5^{\prime \prime} \mathrm{E}, 63^{\circ} 18^{\prime} 01.7^{\prime \prime} \mathrm{N} 36^{\circ} 41^{\prime} 12.9^{\prime \prime} \mathrm{E}$, $63^{\circ} 30^{\prime} 51.1^{\prime \prime N} 36^{\circ} 37^{\prime 2} 2.2^{\prime} \mathrm{E}$, Smyr, Aher, Sroc, Sbir, 11.06.2011, 07.06.2018, leg. VT, PZV; $63^{\circ} 21^{\prime} 05.6^{\prime \prime} \mathrm{N} 36^{\circ} 43^{\prime} 27.7^{\prime \prime} \mathrm{E}, 63^{\circ} 19^{\prime} 33.4$ "N 36²'40.4”E, Basp, 01-06.07.2016, leg. TP, SYKO.

PHYSCIA DUBIA (Hoffm.) Lettau - on bark of wil-

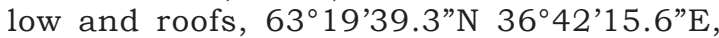
AbVi, 16.06.2011, leg. VT, PZV; 63⒉ $21^{\prime} 02.1^{\prime \prime} \mathrm{N}$ 3643’30.7”E, 6319'45.9”N 3642'17.5” E, Psb, on boulders, bark of willow, 01-05.07.2016, leg. TP, SYKO.

Physcia stellaris (L.) Nyl. - on bark of willow and aspen, 6319'39.3" $\mathrm{N} 36^{\circ} 42^{\prime} 15.6^{\prime \prime} \mathrm{E}$, AbVi, 16.06.2011, leg. VT, PZV; 6319'45.9”'N 3642'17.5”E, AbVi, 01.07.2016, leg. TP, SYKO.

! Physcia tenella (Scop.) DC. - on bark of as-

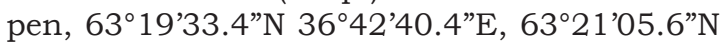
3643’27.7”E, Basp, 01-02.07.2016, leg. TP, SYKO.
Physconia distorta (With.) J. R. Laundon. - on bark of aspen, $63^{\circ} 23^{\prime} 47.1^{\prime \prime} \mathrm{N} 36^{\circ} 36^{\prime} 43.1^{\prime \prime} \mathrm{E}$, $63^{\circ} 23^{\prime} 47.1^{\prime \prime N} 36^{\circ} 36^{\prime} 43.1^{\prime \prime} \mathrm{E}$, Bher, Aher, 14.06.2011, leg. VT, PZV.

Placynthiella oligotropha (J. R. Laundon) Coppins \& P. James - on stone with thin soil layer, 6330'51.1"N 36³7'28.2”E, Sbir, Sroc, 08.06.2018, leg. VT, PZV.

! Polycauliona candelaria (L.) Frödén et al. - on bark of willow and worked timber, 63 $19^{\prime} 39.3^{\prime \prime} \mathrm{N}$ 36 42'15.6” E, AbVi, 16.06.2011, leg. VT, PZV; $63^{\circ} 19^{\prime} 45.9^{\prime \prime} \mathrm{N} 36^{\circ} 42^{\prime} 17.5^{\prime \prime} \mathrm{E}, 63^{\circ} 19^{\prime} 45.9^{\prime \prime} \mathrm{N}$ 36²'17.5”'E, AbVi, 01-05.07.2016, leg. TP, SYKO.

! Polycauliona polycarpa (Hoffm.) Frödén et al.

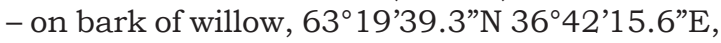
AbVi, 16.06.2011, leg. VT, PZV.

! Porpidia macrocarpa (DC) Hertel \& A.J. Schwab - on stones and boulders, $63^{\circ} 34^{\prime} 14.4^{\prime \prime} \mathrm{N}$ 3661'40.2"E, Proc, R, 13.06.2011, leg. AS, PZV.

! PRotopannaria Pezizoides (Weber) P. M. Jørg. \& S. Ekman - at trunk base of spruce, on bark of aspen, 6320'53.6” $\mathrm{N} 36^{\circ} 33^{\prime} 18.5^{\prime \prime} \mathrm{E}, 63^{\circ} 30^{\prime} 08.0^{\prime \prime} \mathrm{N}$ 36³8'20.5”E, Smyr, 24.06.2018, leg. AV, PZV.

! PycnoRA XANTHOCOCCA (Sommerf.) Hafellner - on bark of aspen, $63^{\circ} 23^{\prime} 47.1^{\prime \prime N} 36^{\circ} 36^{\prime} 43.1^{\prime \prime} \mathrm{E}$, Sher, 15.06.2011, leg. VT, PZV.

! Pseudothelomma ocellatum (Körb.) M. Prieto \& Wedin - on worked timber, 6319'39.3"N 3642'15.6”'E, AbVi, 16.06.2011, leg. VT, PZV.

PSOROMA HYPNORUM (Vahl) Gray - on decaying wood at trunk base of juniper, $63^{\circ} 20^{\prime} 24.3^{\prime \prime} \mathrm{N}$ 3643’22.9”E, Bsph, 05.07.2016, leg. TP, SYKO.

! Racodium Rupestre Pers.: Fr. - on mossy boulders and vertical rocks, 63 ${ }^{\circ} 16^{\prime} 15.06$ "N 36³9'49.26”E, R, 07.06.2016, leg. ECh, det. D. Himelbrant, SYKO.

! Ramboldia cinnabarina (Sommerf.) Kalb et al. - on bark of standing deadwood of spruce, 6330'51.1"N 36³7'28.2”E, Sbir, Sroc, 11.06.2018, leg. VT, PZV.

! Ramboldia Elabens (Fr.) Kantvilas \& Elix - on

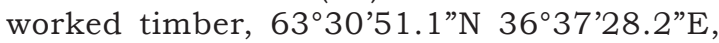
Sbir, Sroc, 11.06.2018, leg. VT; 74³0'54.6”'N 47³7’20.3”E, Sbir, 24.06.2018, leg. AV, PZV.

Ramalina dilacerata (Hoffm.) Hoffm. - on bark of willow and aspen, $63^{\circ} 23^{\prime} 47.1^{\prime \prime} \mathrm{N}$

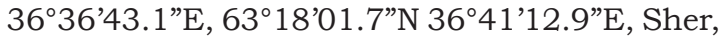


Aher, 15.06.2011, leg. VT, PZV; 63¹9'33.4”N 3642'40.4”E, Basp, 01.07.2016, leg. TP, SYKO.

RAMALINA FARINACEA (L.) Ach. - on bark of aspen and willow, 63 $18^{\prime} 01.7^{\prime \prime} \mathrm{N} 36^{\circ} 41^{\prime} 12.9^{\prime \prime} \mathrm{E}$, Aher, 16.06.2011, leg. VT, PZV; 63⒉ $21^{\prime} 05.6^{\prime \prime} \mathrm{N}$

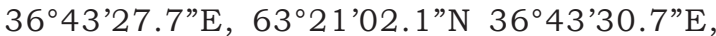
$63^{\circ} 17^{\prime} 58.4^{\prime \prime} \mathrm{N} 36^{\circ} 40^{\prime} 55.2$ "E, Basp, Psb, Aher, 01-03.07.2016, leg. TP, SYKO.

RAMALINA SINENSIS Jatta - on bark of willow, 6318'01.7'N 3641'12.9" E, Aher, 16.06.2011, leg. VT, PZV; on bark of aspen, 6319'33.4”N 3642'40.4”E, Basp, 05.07.2016, leg. TP, SYKO.

! RHizocarpon ferax H. Magn. - on boulders, 6330'51.1"N 36³7'28.2"E, Sbir, Sroc, 11.06.2018, leg. AS, PZV.

! RHIZOCARPON OBSCURATUM (Ach.) A. Massal. - on rocks and stones, 6334'14.4"N 36 61'40.2” E, Proc, R, 13.06.2011, leg. AS, PZV.

! RHIZOCARPON RUBESCENS Th. Fr. - on boulders, 6330'51.1"N 36³7'28.2"E, Sbir, Sroc, 11.06.2018, leg. AS, PZV.

! Rhizoplaca CHRYSOleuca (Sm.) Zopf - on boul-

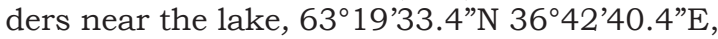
Basp, 01.07.2016, leg. TP, SYKO.

! Rinodina aRchaea (Ach.) Arnold - on worked timber, 6319'39.3” N 36²'15.6” E, AbVi, 16.06.2011, leg. VT, PZV.

! RINODINA PYRINA (Ach.) Arnold - on bark of aspen, 6319'39.3” N 3642'15.6” E, AbVi, 16.06.2011, leg. VT, PZV.

! RinOdina SEPTENTRIONALIS Malme - on bark of alder, $63^{\circ} 23^{\prime} 47.1^{\prime \prime N} 36^{\circ} 36^{\prime} 43.1$ "E, Sher, 15.06.2011, leg. VT, PZV.

! Rinodina SOPHODES (Ach.) A. Massal. - on bark o willow, 6319'39.3” N 3642'15.6” E, AbVi, 16.06.2011, leg. VT, PZV.

! RopalOSPORA VIRIDIS (Tønsberg) Tønsberg - on bark of willow, 63³0'51.1"N 36³7'28.2"E, Sbir, Sroc, 11.06.2018, leg. VT, PZV.

! Rostania occultata (Bagl.) Otálora et al. on bark of aspen and willow, $63^{\circ} 23^{\prime} 47.1^{\prime \prime} \mathrm{N}$

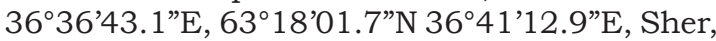
Aher, 15-16.06.2011, leg. VT; 6329'55.1"N 36³9'07.4"E, Smyr, 23.06.2018, leg. AV, PZV;

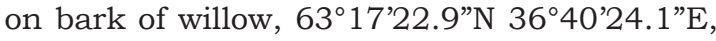
Pspr, 06.07.2016, leg. TP, SYKO.

! Sclerophora CONIOPHAEA (Norman) J. Mattsson \& Middelb. - on wood of birch stump,
6319'33.09”N 36³9'10.0”E, Bher, 03.07.2016, leg. TP, SYKO.

! Scoliciosporum umbrinum (Ach.) Arnold. - on

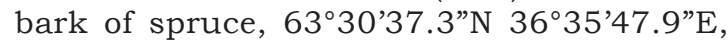
Smyr, 11.06. leg. AV, leg. AV, PZV.

Scutula CIRCUMSPECTA (Vain.) Kistenich et al. - on bark of willow, 6330'51.1"N 36³7'28.2"E, Sbir, Sroc, 09.06.2018, leg. VT, PZV.

! Scytinium Subtile (Schrad.) Otálora et al. - on bark of aspen, 6320'53.6” N 36 33'18.5” E, 6319'54.5” N 36³4'49.0"E, Smyr, Proc, 14.06.2011, leg. VT, PZV.

! SCYTinium TERETIUSCULUm (Wallr.) Otálora et al. - on bark of aspen, $63^{\circ} 20^{\prime} 53.6^{\prime \prime} \mathrm{N} 36^{\circ} 33^{\prime} 18.5^{\prime \prime} \mathrm{E}$, $63^{\circ} 18^{\prime} 01.7^{\prime \prime N} 36^{\circ} 41^{\prime} 12.9^{\prime \prime E}$, Smyr, Aher, 14.06.2011, leg. VT; 63³0’39.9”N 36³7’20.9”'E, Smyr, 13.06.2017, leg. AV, PZV.

Stenocybe pullatula (Ach.) Stein - on bark of alder, $63^{\circ} 23^{\prime} 47.1^{\prime \prime N} 36^{\circ} 36^{\prime} 43.1^{\prime \prime} \mathrm{E}$, Sher, 15.06.2011, leg. VT, PZV.

STEREOCAUlon alPinum Laurer - on mossy boulders, 6320'40.3”N 3643'24.6” E, FR, 02.07.2016, leg. TP, SYKO.

! Stereocaulon Dactylophyllum Flörke - on mossy boulders, $63^{\circ} 17^{\prime} 18.6^{\prime \prime} \mathrm{N} 36^{\circ} 39^{\prime} 50.4^{\prime \prime} \mathrm{E}$, $63^{\circ} 17^{\prime} 09.3^{\prime \prime} \mathrm{N} 36^{\circ} 39^{\prime} 34.7$ "E, Plich, 06.07.2016, leg. TP, SYKO.

Stereocaulon subCORALLOIDES (Nyl.) Nyl. - on stones and mossy boulders, 63 30'51.1"N 36³7'28.2"E, Sbir, Sroc, 11.06.2018, leg. AS, PZV; 6316'15.06”N 36³9'49.26” E, R, leg. ECh, det. TP, 631'18.6”N 36³9'50.4”E, Proc, 06.07.2016, leg. TP, SYKO.

STRANGOSPORA MORIFORMIS (Ach.) Stein - on

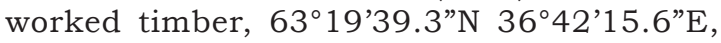
AbVi, 16.06.2011, leg. VT, PZV.

! Toensbergia leucococca (R. Sant.) Bendiksby $\&$ Timdal - on bark of deciduous and coniferous trees, mainly on spruce branches of spruce, 6330'51.1'N 36³7'28.2”'E, Sbir, Sroc, 11.06.2018, leg. VT; 63³0’51.2”N 36³7'36.1”'E, 24.06.2018, leg. AV, PZV; on bark of rowan, 63¹9'19.6” N 3643'12.2”'E, Spin, 03.07.2016, leg. TP, SYKO.

TONINIOPSIS SUBINCOMPTA (NYL.) Kistenich et al. - on bark of deciduous trees, $63^{\circ} 20^{\prime} 53.6^{\prime \prime} \mathrm{N}$ $36^{\circ} 33^{\prime} 18.5^{\prime \prime} \mathrm{E}, 63^{\circ} 23^{\prime} 47.1^{\prime \prime} \mathrm{N} 36^{\circ} 36^{\prime} 43.1^{\prime \prime} \mathrm{E}$, $63^{\circ} 19^{\prime} 39.3^{\prime \prime} \mathrm{N} 36^{\circ} 42^{\prime} 15.6^{\prime \prime} \mathrm{E}, 63^{\circ} 18^{\prime} 01.7^{\prime \prime} \mathrm{N}$ $36^{\circ} 41^{\prime} 12.9^{\prime \prime} E$, Smyrt, Sher, Aher, AbVi, 14- 
16.06.2011, leg. VT, PZV; on bark of aspen, $63^{\circ} 17^{\prime} 58.4$ "N 3640'55.2”'E, Aher, 03.07.2016, leg. TP, SYKO.

! Xanthoparmelia stenophylla (Ach.) Ahti \& D. Hawksw. - on stones and boulders, 63 $30^{\circ} 51.1^{\prime \prime} \mathrm{N}$ 36³7'28.2”E, Sbir, Sroc, 11.06.2018, leg. AS, PZV.

XANTHORia PARietina (L.) Th. Fr. - on bark of as-

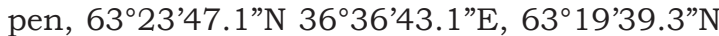
$36^{\circ} 42^{\prime} 15.6^{\prime \prime} \mathrm{E}, 63^{\circ} 18^{\prime} 01.7^{\prime \prime} \mathrm{N} 36^{\circ} 41^{\prime} 12.9^{\prime \prime} \mathrm{E}$, Sher, AbVi, Aher, 15.06.2011, leg. VT, PZV; $63^{\circ} 21^{\prime} 17.2$ "N 3643'24.6”'E, Psb, 01.07.2016, leg. TP, SYKO.

XYLOGRAPHA PARAllela (Ach.: Fr.) Fr. - on decaying wood and timber, $63^{\circ} 23^{\prime} 47.1^{\prime \prime} \mathrm{N} 36^{\circ} 36^{\prime} 43.1^{\prime \prime} \mathrm{E}$, $63^{\circ} 30^{\prime} 51.1^{\prime \prime N} 36^{\circ} 37^{\prime} 28.2^{\prime \prime E}$, Sher, Sbir, Sroc, 15.06.2011, 08.06.2018, leg. VT, PZV.

! XYlogRAPHA RUBESCENS Räsänen - on decaying wood and timber, 63 $30^{\prime} 51.1^{\prime \prime} \mathrm{N} 36^{\circ} 37^{\prime} 28.2^{\prime \prime} \mathrm{E}$, Sbir, Sroc, 11.06.2018, leg. VT; 70³0’54.6”N 43³5’47.9”'E, Sbir, 24.06.2018, leg. AV, PZV.

\section{DISCUSSION}

In total, 155 species of lichens and allied fungi were recorded for the first time for Arkhangelsk part of Vodlozersky National Park. Thus, 342 and 3 subspecies of lichens are currently known from the Arkhangelsk part of the Park (Tarasova et al., 2016).

The species Cladonia bellidiflora, included in the Red Data Book of Arkhangelsk Region (2008), was recorded for the first time in Arkhangelsk part of the Park. In the neighboring territory of Karelia which has been studied much better, eight species of the presented list are regionally protected (Red Data Book of Karelian Republic, 2007): Bryoria bicolor, B. nadvornikiana, Chaenotheca stemonea, Hypogymnia bitteri, Nephroma bellum, Ramalina dilacerata, Sclerophora coniophaea and Stereocaulon dactylophyllum; five of these species are included also in the Red Data Book of East Fennoscandia (Kotiranta et al., 1998): Bryoria bicolor, B. nadvornikiana, Chaenotheca stemonea, Sclerophora coniophaea and Stereocaulon dactylophyllum.

In the list 69 species are new for the mainland area of the Arkhangelsk Region. Altogether 514 lichens and allied fungi are known nowadays for the mainland area of the Arkhangelsk Region, taking into consideration data on 445 species known from the region before (Tarasova et al., 2015, 2016).

The results of the presented study demonstrate significant potential of the lichen diversity in the territory of Vodlozersky National Park including its Arkhangelsk part.

\section{ACKNOWLEDGEMENTS}

We would like to express our gratitude to Orvo Vitikainen for confirmation of Peltigera specimens and the staff of Vodlozersky National Park (Viktor Mamontov, Maria Shreders, Vasily Ruchyovskih \& Sergei Zakutin) for help in organizing the expedition to the localities hard to reach; D. E. Himelbrant and I. S. Stepanchikova are thanked for assistance in the identification of some taxa and consultations. Thanks are given to Elena Yu. Churakova for collections of lichen specimens. We are grateful to the editors and reviewers for valuable corrections and recommendations which have improved the quality of our work.

The study was supported by the Ministry of Education and Science of the Russian Federation (project No 5.8740.2017/k); additional support was given by the Institute of Biology of Komi Scientific Centre of the Ural Branch and Avrorin Polar-Alpine Botanical Garden-Institute of the Russian Academy of Sciences (institutional projects No AAAA-A19-119011790022-1 and AAAA-A18-118050490088-0) and administration of Vodlozersky National Park.

\section{REFERENCES}

Androsova, V., Tarasova, V. \& Gorshkov, V. 2018. Diversity of lichens and allied fungi on Norway spruce (Picea abies) in the middle boreal forests of Republic of Karelia (Russia). Folia Cryptogamica Estonica 55: 133-149. https://doi.org/10.12697/ fce. 2018.55 .14

Fadeeva, M. A. 2005. Nemoral species in the flora of lichens of Karelia. Biogeography of Karelia. Proceedings of Karelian Research Centre of RAS 7: 255-265. (In Russian).

Fadeeva, M. A., Golubkova, N. S., Vitikainen, O. \& Ahti, T. 2007. Conspectus of lichens and lichenicolous fungi of the Republic of Karelia. Petrozavodsk, 194 pp. (In Russian).

Golubkova, N. S. 2003. Bacidina. In: Handbook of Lichens of Russia 8: 40-46. (In Russian). 
Ignatenko, R., \& Tarasova, V. 2017. The population structure of the lichen Lobaria pulmonaria in the middle boreal forests depends on the time-sincedisturbance. Folia Cryptogamica Estonica 54: 83-94. https://doi.org/10.12697/fce.2017.54.13

Kotiranta, H., Uotila, P., Sulkava, S. \& Peltonen, S.-L. (eds) 1998. Red Data Book of East Fennoscandia. 1998. Helsinki. 351 pp.

Nordin, A., Moberg, R., Tønsberg, T., Vitikainen, O., Dalsätt, Å., Myrdal, M., Snitting, D. \& Ekman, S. 2011. Santesson's Checklist of Fennoscandian Lichen-forming and Lichenicolous Fungi. Ver. April 29, 2011. http://130.238.83.220/santesson/ home.php (25 March 2019).

Orange, A., James, P. W. \& White, F. J. 2001. Microchemical methods for the identification of lichens. British Lichen Society, London. 101 pp.

Red Data Book of Arkhangelsk Region. 2008. Arkhangelsk. 351 pp. (In Russian).

Red Data Book of Karelian Republic. 2007. Petrozavodsk. 368 pp. (In Russian).

Tarasova, V., Obabko, R., Himelbrant, D., Boychuk, M., Stepanchikova, I. \& Borovichev, E. 2017. Diversity and distribution of epiphytic lichens and bryophytes on aspen (Populus tremula) in the middle boreal forests of Republic of Karelia (Russia). Folia Cryptogamica Estonica 54: 125-141. https://doi.org/10.12697/fce.2017.54.16

Tarasova, V., Sonina, A., Androsova, V. \& Stepanchikova, I. 2015. The lichens of forest rocky communities of mountain Olovgora (Arkhangelsk Region, Northwest Russia). Folia Cryptogamica Estonica 52: 51-62. https:/doi.org/10.12697/ fce.2015.52.07

Tarasova, V., Sonina, A., Androsova, V. \& Stepanchikova, I. 2016. The lichens of forest rocky communities of the hill Muroigora (Arkhangelsk Region, Northwest Russia). Folia Cryptogamica Estonica 53: 111-121. https://doi.org/10.12697/ fce.2016.53.13

Tarasova, V. N. \& Stepanchikova I. S. 2016. New lichens to Karelia Republic. Proceedings of Petrozavodsk State University. Biological sciences 4(157): 78-82. (In Russian).

Tarasova, V. N. \& Stepanova, V. I. 2001. Preliminary list of lichens of the National Park "Vodlozersky". In: National Park "Vodlozersky": the natural diversity and cultural heritage. Petrozavodsk. Pp. 183-192. (In Russian). 